\title{
Luftverschmutzung und Gesundheit
}

Durch die Luftverschmutzung wird nicht nur der Wald, sondern auch die menschliche Gesundheit in zunehmendem Mass gefährdet. Luftschadstoffe können vor allem die Anfälligkeit für Atemwegserkrankungen erhöhen und deren Verlauf ungünstig beeinflussen. Besonders empflindlich reagieren Kinder sowie kranke und alte Menschen. Dies sind die wichtigsten Schlussfolgerungen der Tagung über «Luftverunreinigung und Gesundheit», die am 7. und 8. November 1985 an der Eidg. Technischen Hochschule in Zürich von der Schweizerischen Gesellschaft für Sozial- und Präventivmedizin in Zusammenarbeit mit der Schweizerischen Vereinigung gegen Tuberkulose und Lungenkrankheiten und der Schweizerischen Gesellschaft für Pneumologie durchgeführt wurde. Die Tagung stand unter dem Patronat von Herrn Bundesrat Dr. A. Egli, Vorsteher des Eidg. Departementes des Innern, und Dr. med. K. Zimmermann, Präsident der Vereinigung der Schweizer Ärzte.

Das vorliegende Sonderheft enthält alle anlässlich dieser Tagung gehaltenen Referate. Mit den am 1. Tag (Informationstagung) vorgetragenen Berichten wurde eine Übersicht über die heute bekannten gesundheitlichen Auswirkungen von Luftschadstoffen gegeben. Schwerpunkte bildeten dabei die Ergebnisse epidemiologischer Untersuchungen, die Bewertung akuter und chronischer Schadstoffbelastungen sowie die Beurteilung der Immissionssituation in der Schweiz und die sich daraus ergebenden Konsequenzen. Am 2. Tag (Wissenschaftliche Tagung) standen die zur Zeit in der Schweiz laufenden Studien und Immissionsmessungen im Mittelpunkt. Dabei zeigte sich sehr deutlich, dass noch zahlreiche Lücken bestehen. Detaillierte statistische Angaben über die Erkrankungen, die in einem Zusammenhang mit der Luftverschmutzung stehen können, liegen noch nicht vor. Hinweise dazu gibt jedoch der in den letzten Jahren zunehmende Trend von Bronchial- und Lungenkrankheiten. Aussagen über die Häufigkeit und Schwere von Atemwegserkrankungen bei Kindern werden aufgrund der Erhebungen möglich sein, die im kommenden Jahr in Basel und Zürich durchgeführt werden.

Anlässlich der Tagung wurde wiederholt betont, dass die bisher in der Schweiz getroffenen Massnahmen zur Reduktion der Luftverschmutzung ungenügend sind. Die Schadstoffbelastungen in den Städten, Agglomerationen und auch in ländlichen Gegenden sind zum Teil sehr hoch. Die aus gesundheitlicher Sicht sowie zum Schutz der Umwelt noch als zumutbar beurteilten Immissionen werden sehr oft überschritten. In einer von den Tagungsteilnehmern verabschiedeten Resolution werden deshalb insbesondere folgende Massnahmen als vordringlich erachtet: Inkraftsetzung der Luftreinhalteverordnung durch den Bundesrat und ein strenger Vollzug derselben durch die Kantone, Erlass von wirksamen Vorschriften zur Begrenzung des Schadstoffausstosses von Dieselfahrzeugen, sowie Einschränkungen des individuellen motorisierten Verkehrs durch weiteren Ausbau eines attraktiven Angebotes des öffentlichen Verkehrs.

H. U. Wanner 


\section{La pollution de l'air et santé}

La pollution atmosphérique met en péril non seulement nos forêts mais aussi notre santé, et ceci, dans une mesure toujours plus grande. C'est ainsi que les polluants de l'air ont en particulier la propriété d'augmenter notre vulnérabilité envers les atteintes respiratoires et influencent défavorablement leur évolution. Les enfants, les malades, les personnes âgées sont les plus menacées.

Voici, en quelques mots, les conclusions principales auxquelles sont parvenues les journées d'information sur «la pollution de l'air et la santé» qui ont eu lieu, le 7 et 8 novembre derniers, à l'Ecole polytechnique fédérale de Zürich. Organisées par la Société suisse de médecine sociale et préventive, en collaboration avec l'Association suisse contre la tuberculose et les maladies pulmonaires, d'une part, et la Société suisse de pneumologie, d'autre part, cette réunion a été placée sous le patronage du Conseiller fédéral A. Egli et du Dr. K. Zimmermann, président de la Fédération des médecins suisses.

Le présent numéro réunit les textes complets des interventions qui ont été faites à ces journées, la première ayant été consacrée aux connaissances que l'on a aujourd'hui des effets de la pollution de l'air sur la santé ainsi que les moyens de les mesurer. La seconde, dite «journée scientifique» a été dévolue essentiellement aux travaux réalisés actuellement en Suisse dans ce domaine.

Comme bilan de ces communications, on peut prétendre que nos connaissances scientifiques sont encore insuffisantes et qu'on y trouve de nombreuses lacunes. En particulier, les données statistiques suisses sur les maladies qui pourraient découler de l'exposition à la pollution atmosphérique sont encore peu nombreuses. Il a été mentionné comme un fait inquiétant que les affections broncho-pulmonaires sont en augmentation dans notre pays. On attend avec impatience les résultats d'une vaste enquête sur les enfants qui est actuellement conduite à Bâle et à Zürich.

De plus, il a été souligné à plusieurs reprises que les niveaux de pollution mesurés dans nos villes, nos bourgades et même dans nos campagnes dépassaient souvent les limites admissibles. Les mesures qui devraient être prises pour réduire ces immissions nocives sont largement insuffisantes.

Dans une résolution émise par les participants à ces journées, les actions de prévention qui sont considérées comme les plus urgentes ont été énumérées. Il s'agit de:

- la promulgation de l'ordonnance sur la lutte contre la pollution atmosphérique,

- l'exécution stricte de cette ordonnance par les cantons,

- la mise au point d'un règlement destiné à réduire la pollution par les moteurs Diesel,

- un programme de réduction du trafic motorisé individuel par la mise en service des moyens de transport collectif attractifs.

H. U. Wanner 